\title{
Some Remarks on PDOP and TDOP for Multi-GNSS Constellations
}

\author{
Yunlong Teng ${ }^{1}$ and Jinling Wang ${ }^{2}$ \\ ${ }^{1}$ (School of Energy Science and Engineering, University of Electronic Science and \\ Technology of China, Chengdu, Sichuan Province, 611731, PR China) \\ ${ }^{2}$ (School of Civil and Environmental Engineering, University of New South Wales, \\ Sydney, NSW 2052, Australia) \\ (E-mail: ylteng@uestc.edu.cn)
}

\begin{abstract}
For single point positioning solutions with multi-Global Navigation Satellite System (GNSS) constellations, the Position Dilution Of Precision (PDOP) and Time Dilution Of Precision TDOP) are quality measures to specify the additional multiplicative affect of measurement error on positioning accuracy and the timing accuracy, respectively. Considering the dimension of the unknown vector (including the three-dimensional positioning information of the receiver as well as the receiver clock biases related to these single constellations), this paper theoretically derives new formulae to describe the change of PDOP and TDOP. In addition, the detailed expressions of the variable quantity of the PDOP and TDOP are also obtained. The results show that if the dimension of the unknown vector is invariant in case of removing one or more satellites belonging to the existing tracked multi-GNSS constellations, both the PDOP and TDOP increase. On the other hand, if removing satellites reduces the dimension of the unknown vector, the PDOP neither increases nor decreases. However, the change of the TDOP is different, and it becomes smaller.
\end{abstract}
KEYWORDS
1. Satellite Navigation.
2. GNSS.
3. Constellation.
4. Dilution of Precision.

Submitted: 25 July 2014. Accepted: 16 June 2015. First published online: 3 July 2015.

1. INTRODUCTION. The future of Global Navigation Satellite Systems (GNSS) is promising, with the integration of multi-GNSS constellations, such as the Global Positioning system (GPS), Globalnaya Navigatsionnaya Sputnikovaya Sistema (GLONASS), Galileo and BeiDou System (BDS or Compass), becoming an important direction for future development of satellite navigation (Blomenhofer, 2004; Shi and Cui, 2011). In comparison with single constellations, multi-GNSS constellations will be beneficial for improvements of positioning calculation and integrity monitoring (Hewitson and Wang, 2006; Wang and Ober, 2009; Teng and Shi, 2012; Xu et al., 2012; Angrisano et al., 2013; Dautermann, 2014; Torre and Caporali, 2015).

The Geometric Dilution of Precision (GDOP) is an important parameter utilised in the propagation of random error (noise) in measurements of the noise levels of the unknown (solved for) parameters (Yang et al., 2011), and it is a quality measure to 
specify the additional multiplicative affect of measurement error on the positioning accuracy and the timing accuracy. Actually, the GDOP can be divided into the PDOP (Position Dilution Of Precision) and (Time Dilution Of Precision). The former describes the effect of geometry on the relationship between the measurement error and the three-dimensional positioning error. The latter is related to the timing accuracy. The lower PDOP and TDOP values are, the higher positioning accuracy and the timing accuracy will be, given the same level of measurement error (Kaplan and Hegarty, 2006; Rzepecka et al., 2014).

In our former studies (Teng and Wang, 2014), the change of GDOP with the number of satellites was discussed. In this paper, we mainly analyse the change of PDOP and TDOP with respect to the number of satellites. The remaining parts of this paper are organised as follows. The definitions of PDOP and TDOP with multi-GNSS constellations are given in Section 2. The relationship between them and the number of satellites are derived theoretically in Section 3. Moreover, the detailed expressions of the variable quantity of the PDOP and TDOP are also obtained in this section. In Section 4, a numerical experiment will be provided to validate the change of PDOP and TDOP. Finally, some conclusions are drawn.

\section{DEFINITION OF PDOP AND TDOP IN MULTI-GNSS CONSTELLA-} TIONS. For single point positioning with multi-GNSS constellations, the coordinate and time system errors between different single constellations are two important issues to be solved. Yang et al. (2011) analysed the coordinate system errors, and concluded that the difference of coordinate systems used in the GNSS constellations has no influence on the calculation of GDOP, PDOP and TDOP.

In terms of dealing with the differences in the time systems, there are generally two ways (Kaplan and Hegarty, 2006; Defraigne and Baire, 2011). One is broadcasting the time difference between different constellations in the broadcast ephemeris. Such observed time differences (called GNSS time offsets) may also be treated as pseudomeasurements (Wang et al., 2011). The other is adding one unknown time system error parameter. In this paper, the time difference is considered as one unknown parameter in the process of positioning calculation.

When the GNSS positioning is based on one single constellation, for example GPS (the coordinate reference framework WGS-84), the following pseudorange equation between the $i$ th satellite and the receiver is given by

$$
p_{i}=\left\|\boldsymbol{r}-\boldsymbol{r}_{i}\right\|+c \Delta t_{1}
$$

where $\boldsymbol{r}=(x, y, z)$ denotes the three-dimensional coordinates of the receiver, $p_{i}$ and $\boldsymbol{r}_{i}=$ $\left(x_{i}, y_{i}, z_{i}\right)$ denotes the pseudorange and the three-dimensional coordinates of the $i$ th satellite, respectively, $c \Delta t_{1}$ is the receiver clock bias relative to the constellation in unit of meter. In Equation (1), the detailed procedures for calculating the position of each satellite $\left(\boldsymbol{r}_{i}\right)$ can be found in Rapinski et al. (2012).

In the process of positioning calculation, a truncated Taylor Series expansion is applied to Equation (1) around the approximate coordinates of the receiver $\overrightarrow{\boldsymbol{r}}=(\vec{x}, \vec{y}, \vec{z})$,

$$
\Delta p_{i}=p_{i}-\vec{p}_{i}=\boldsymbol{h}_{i} \Delta \boldsymbol{r}^{T}+c \Delta t_{1}
$$


where $\vec{p}_{i}=\left\|\overrightarrow{\boldsymbol{r}}-\boldsymbol{r}_{i}\right\|, \Delta \boldsymbol{r}=\boldsymbol{r}-\overrightarrow{\boldsymbol{r}}$, and $\boldsymbol{h}_{i}=\left(\overrightarrow{\boldsymbol{r}}-\boldsymbol{r}_{i}\right) / \vec{p}_{i} \in R^{1 \times 3}$ denotes the direction cosine vector from the receiver to the $i$ th satellite.

When there are $\alpha$ satellites, the measurement equation for the positioning calculation can be written as

$$
\left[\begin{array}{c}
\Delta p_{1} \\
\Delta p_{2} \\
\cdots \\
\Delta p_{\alpha}
\end{array}\right]=\left[\begin{array}{cc}
\boldsymbol{h}_{1} & 1 \\
\boldsymbol{h}_{2} & 1 \\
\ldots & \ldots \\
\boldsymbol{h}_{\alpha} & 1
\end{array}\right]\left[\begin{array}{ll}
\Delta \boldsymbol{r} & c \Delta t_{1}
\end{array}\right]^{T}
$$

which has a general form represented as

$$
\boldsymbol{z}_{1}=\boldsymbol{H}_{1} \Delta \boldsymbol{x}_{1}
$$

In Equation (4), $z_{1}$ represents the measurement vector, $\Delta \boldsymbol{x}_{1}=\left[\begin{array}{ll}\Delta \boldsymbol{r} & c \Delta t_{1}\end{array}\right]^{T}$ denotes the unknown vector to be estimated and it includes four unknown parameters. The design matrix $\boldsymbol{H}_{1}$ is given by

$$
\boldsymbol{H}_{1}=\left[\begin{array}{cccc}
\boldsymbol{h}_{1}^{T} & \boldsymbol{h}_{2}^{T} & \cdots & \boldsymbol{h}_{\alpha}^{T} \\
1 & 1 & \cdots & 1
\end{array}\right]^{T}=\left[\begin{array}{ll}
\vec{H}_{1} & \boldsymbol{1}_{\alpha}
\end{array}\right]
$$

Similarly, if one or more constellations are combined with the GPS constellation, the corresponding measurement equation is expressed as

$$
\left\{\begin{array}{l}
\boldsymbol{z}_{2}=\boldsymbol{H}_{2} \Delta \boldsymbol{x}_{2}=\left[\begin{array}{ll}
\vec{H}_{2} & \boldsymbol{1}_{\beta}
\end{array}\right]\left[\begin{array}{ll}
\Delta \boldsymbol{r} & c \Delta t_{2}
\end{array}\right]^{T} \\
\boldsymbol{z}_{3}=\boldsymbol{H}_{3} \Delta \boldsymbol{x}_{3}=\left[\begin{array}{ll}
\vec{H}_{3} & \boldsymbol{1}_{\gamma}
\end{array}\right]\left[\begin{array}{ll}
\Delta \boldsymbol{r} & c \Delta t_{3}
\end{array}\right]^{T} \\
\cdots
\end{array}\right.
$$

where the design matrices $\boldsymbol{H}_{2}, \boldsymbol{H}_{3}, \cdots$ have $\beta, \gamma, \cdots$ rows, respectively. The number of rows equals the number of tracked satellites in the respective constellations. The $c \Delta t_{2}, c \Delta t_{3}, \cdots$ are the receiver clock biases associated with these constellations.

Combining Equation (6) with Equation (4) leads to

$$
\boldsymbol{z}=\boldsymbol{H} \Delta \boldsymbol{x}
$$

where

$$
\left\{\begin{array}{l}
\boldsymbol{z}=\left[\begin{array}{llll}
\boldsymbol{z}_{1}^{T} & \boldsymbol{z}_{2}^{T} & \boldsymbol{z}_{3}^{T} & \cdots
\end{array}\right]^{T} \\
\Delta \boldsymbol{x}=\left[\begin{array}{lllll}
\Delta \boldsymbol{r} & c \Delta t_{1} & c \Delta t_{2} & c \Delta t_{3} & \cdots
\end{array}\right]^{T}
\end{array}\right.
$$

Suppose the multi-GNSS constellations are composed of $m$ individual constellations, the number of receiver clock bias equals $m$. Thus, there are $(m+3)$ parameters to be calculated in the unknown vector. In addition, the design matrix $\boldsymbol{H}$ is given by

$$
\boldsymbol{H}=\left[\begin{array}{lllll}
\overrightarrow{\boldsymbol{H}}_{1} & \boldsymbol{1}_{\alpha} & \boldsymbol{0}_{\alpha} & \boldsymbol{0}_{\alpha} & \cdots \\
\overrightarrow{\boldsymbol{H}}_{2} & \boldsymbol{0}_{\beta} & \boldsymbol{1}_{\beta} & \boldsymbol{0}_{\beta} & \cdots \\
\overrightarrow{\boldsymbol{H}}_{3} & \boldsymbol{0}_{\gamma} & \boldsymbol{0}_{\gamma} & \boldsymbol{1}_{\gamma} & \cdots \\
\cdots & \cdots & \cdots & \cdots & \cdots
\end{array}\right] \in R^{(\alpha+\beta+\gamma+\cdots) \times(m+3)}
$$

In Equation (9), the ones and zeros vectors are located in different columns in order to include each receiver clock bias, because the biases for different constellations are 
different (Wang et al., 2011). Moreover, $n=\alpha+\beta+\gamma+\cdots$ denotes the total number of satellites tracked in the multi-GNSS constellations.

As the proper weighting of individual satellites range measurement is required, the weight matrix $\boldsymbol{Q}$ should be introduced. Moreover, $\boldsymbol{Q}$ is a block diagonal matrix and its diagonal element is associated with the corresponding satellite. More details about the weight matrix and the impact of different measurement errors are discussed in BlancoDelgado and Nunes (2010). When $\boldsymbol{Q}$ is taken into consideration, we define

$$
\boldsymbol{R}=\left(\boldsymbol{H}^{T} \boldsymbol{Q H}\right)^{-1}
$$

Then the PDOP and TDOP in the single point positioning with the multi-GNSS constellations are defined as

$$
P D O P=\sqrt{\sum_{i=1}^{3} r(i)}, \quad T D O P=\sqrt{\sum_{i=4}^{m+3} r(i)}
$$

where $r(i)$ denote the diagonal elements of $\boldsymbol{R}$. It is worth mentioning that the definition of TDOP with the multi-GNSS constellations is relative to the number of the single constellations composed of the multi-GNSS constellations. This is different from the single constellations.

\section{CHANGE OF PDOP AND TDOP IN MULTI-GNSS CONSTELLATIONS}

Regarding the positioning applications with GNSS constellations, open sky is the best environment (Li et al., 2010). However, in certain applications, the satellite signals are susceptible to obstructions. Thus one or more satellites cannot be used for positioning calculation or are not visible anymore. In addition, the impact of higher cut-off elevation angle on the number of satellites should be taken into consideration. To take one example, Teunissen et al. (2014) analysed the influence of the cut-off elevation angle on the number of satellites for a combined GPS/BDS constellation. The results showed that the receiver can track more satellites under a lower cut-off elevation angle compared with the higher one. The above situations can be regarded as removing one or more satellites from the tracked multi-GNSS constellations.

When one satellite belonging to the tracked multi-GNSS constellations is removed, the dimension of the unknown vector (influenced directly by the number of the receiver clock biases) may keep invariant or decreasing. The two different cases lead to different changes of PDOP and TDOP with the number of satellites. In this section, we take an example of multi-GNSS constellations including two single ones (short for the dualGNSS constellations hereafter), and then derive the change of PDOP and TDOP with the number of satellites.

3.1. Invariant Dimension of the Unknown Vector. Supposing that $\boldsymbol{H}_{n}$ and $\boldsymbol{Q}_{n}$ are the design and weight matrices, and the design and weight matrices that removed the $n$th satellite from the constellations are $\boldsymbol{H}_{(n-1)}$ and $\boldsymbol{Q}_{(n-1)}$. If the dimension of the unknown vector remains invariant, then we have

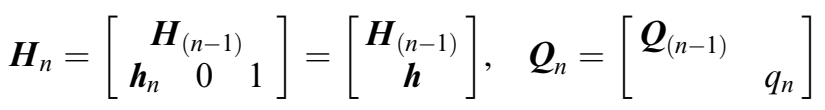

with $\boldsymbol{h}_{n}$ being the direction cosine vector from the receiver to the $n$th satellite. For the 
dual-GNSS constellations, as there are five unknown parameters, the dimensions of $\boldsymbol{H}_{(n-1)}$ and $\boldsymbol{H}_{n}$ are $(n-1) \times 5$ and $n \times 5$, respectively. According to Equation (6), we can obtain

$$
\boldsymbol{H}_{n}^{T} \boldsymbol{Q}_{n} \boldsymbol{H}_{n}=\boldsymbol{H}_{(n-1)}^{T} \boldsymbol{Q}_{(n-1)} \boldsymbol{H}_{(n-1)}+q_{n} \boldsymbol{h}^{T} \boldsymbol{h}
$$

Taking the inverse of both sides of Equation (13) leads to

$$
\boldsymbol{R}_{n}=\left(\boldsymbol{H}_{n}^{T} \boldsymbol{Q}_{n} \boldsymbol{H}_{n}\right)^{-1}=\left(\boldsymbol{H}_{(n-1)}^{T} \boldsymbol{Q}_{(n-1)} \boldsymbol{H}_{n-1}+q_{n} \boldsymbol{h}^{T} \boldsymbol{h}\right)^{-1}
$$

To find the change of PDOP and TDOP with the number of satellites, we consider the Woodbury matrix identity,

$$
(\boldsymbol{A}+\boldsymbol{B C D})^{-1}=\boldsymbol{A}^{-1}-\boldsymbol{A}^{-1} \boldsymbol{B}\left(\boldsymbol{C}^{-1}+\boldsymbol{D} \boldsymbol{A}^{-1} \boldsymbol{B}\right)^{-1} \boldsymbol{D} \boldsymbol{A}^{-1}
$$

where $\boldsymbol{A}, \boldsymbol{B}, \boldsymbol{C}$ and $\boldsymbol{D}$ all denote matrices with the correct size.

From this theorem, we have

$$
\begin{aligned}
\boldsymbol{R}_{n} & =\left(\boldsymbol{H}_{(n-1)}^{T} \boldsymbol{Q}_{(n-1)} \boldsymbol{H}_{n-1}+q_{n} \boldsymbol{h}^{T} \boldsymbol{h}\right)^{-1} \\
& =\left(\boldsymbol{H}_{(n-1)}^{T} \boldsymbol{Q}_{(n-1)} \boldsymbol{H}_{(n-1)}\right)^{-1}-\varphi\left(\boldsymbol{H}_{(n-1)}^{T} \boldsymbol{Q}_{(n-1)} \boldsymbol{H}_{(n-1)}\right)^{-1} \boldsymbol{h}^{T} \boldsymbol{h}\left(\boldsymbol{H}_{(n-1)}^{T} \boldsymbol{Q}_{(n-1)} \boldsymbol{H}_{(n-1)}\right)^{-1} \\
& =\boldsymbol{R}_{n-1}-\varphi \boldsymbol{R}_{(n-1)} \boldsymbol{h}^{T} \boldsymbol{h} \boldsymbol{R}_{(n-1)}
\end{aligned}
$$

where

$$
\varphi=\left[q_{n}^{-1}+\boldsymbol{h}\left(\boldsymbol{H}_{n}^{T} \boldsymbol{Q}_{n} \boldsymbol{H}_{n}\right)^{-1} \boldsymbol{h}^{T}\right]^{-1}=\left[q_{n}^{-1}+\boldsymbol{h} \boldsymbol{R}_{n} \boldsymbol{h}^{T}\right]^{-1}
$$

In Equation (17), $q_{n}^{-1}>0$, and $\boldsymbol{h} \boldsymbol{R}_{n} \boldsymbol{h}^{T}$ is a positive definite quadratic form, so $\varphi$ is also positive.

Let $\boldsymbol{\mu}=\boldsymbol{h} \boldsymbol{R}_{(n-1)} \in R^{1 \times 5}$, then Equation (16) is simplified as

$$
\boldsymbol{R}_{n}=\boldsymbol{R}_{(n-1)}-\varphi \boldsymbol{\mu}^{T} \boldsymbol{\mu}
$$

It is clear that the diagonal elements of the matrix $\boldsymbol{\mu}^{T} \boldsymbol{\mu}$ are positive. Therefore,

$$
r_{n}(i)<r_{(n-1)}(i), \quad i=1, \cdots, 5
$$

Based on the definition of PDOP and TDOP in Equation (11), we obtain

$$
\left\{\begin{array}{l}
\operatorname{PDOP}_{n}<\operatorname{PDOP}_{(n-1)} \\
\operatorname{TDOP}_{n}<\operatorname{TDOP}_{(n-1)}
\end{array}\right.
$$

From the inequalities in Equation (20), it is clear that the PDOP and TDOP always increase when the number of satellites decreases, but at the same time, the dimension of the unknown vector is invariant. Equation (20) is a qualitative analysis about the change of PDOP and TDOP with the number of satellites. 
In addition to deriving the change of PDOP and TDOP, we define

$$
\left\{\begin{array}{l}
\triangle \mathrm{PDOP}_{n}=\left|\mathrm{PDOP}_{n}^{2}-\mathrm{PDOP}_{(n-1)}^{2}\right| \\
\Delta \mathrm{TDOP}_{n}=\left|\mathrm{TDOP}_{n}^{2}-\operatorname{TDOP}_{(n-1)}^{2}\right|
\end{array}\right.
$$

as the variable quantity of the PDOP and TDOP with the number of satellites.

Substituting Equation (16) into Equation (21) leads to

$$
\left\{\begin{array}{l}
\Delta \text { PDOP }_{n}=\varphi \sum_{i=1}^{3}\left[\boldsymbol{R}_{(n-1)}(:, i) \boldsymbol{h}^{T}\right]^{2} \\
\Delta T D O P_{n}=\varphi \sum_{i=4}^{5}\left[\boldsymbol{R}_{(n-1)}(:, i) \boldsymbol{h}^{T}\right]^{2}
\end{array}\right.
$$

where $\boldsymbol{R}_{(n-1)}(:, i)$ denotes the $i$ th row of $\boldsymbol{R}_{(n-1)}$.

Furthermore, substitution of Equation (17) into Equation (22) results in

$$
\left\{\begin{array}{l}
\Delta \mathrm{PDOP}_{n}=\left[q_{n}^{-1}+\boldsymbol{h} \boldsymbol{R}_{n} \boldsymbol{h}^{T}\right]^{-1} \sum_{i=1}^{3}\left[\boldsymbol{R}_{(n-1)}(:, i) \boldsymbol{h}^{T}\right]^{2} \\
\Delta T D O P_{n}=\left[q_{n}^{-1}+\boldsymbol{h} \boldsymbol{R}_{n} \boldsymbol{h}^{T}\right]^{-1} \sum_{i=4}^{5}\left[\boldsymbol{R}_{(n-1)}(:, i) \boldsymbol{h}^{T}\right]^{2}
\end{array}\right.
$$

The equality in Equation (23) describes the variable quantity of the PDOP and the TDOP when the dimension of the unknown vector is invariant. It is a quantitative expression about the change of the PDOP and TDOP.

3.2. Variant Dimension of the Unknown Vector. If removing one satellite from the tracked dual-GNSS constellations reduces the dimension of the unknown vector, it means that there is only one satellite in the first or the second constellation. Herewith we suppose that $\beta=1$, then we have

$$
\overrightarrow{\boldsymbol{H}}_{n}=\left[\begin{array}{ccc}
\overrightarrow{\boldsymbol{H}}_{(n-1)} & 0 \\
\overrightarrow{\boldsymbol{h}}_{n} & 0 & 1
\end{array}\right]=\left[\begin{array}{cc}
\overrightarrow{\boldsymbol{H}}_{(n-1)} & 0 \\
\overrightarrow{\boldsymbol{h}} & 1
\end{array}\right], \quad \overrightarrow{\boldsymbol{Q}}_{n}=\left[\begin{array}{cc}
\overrightarrow{\boldsymbol{Q}}_{(n-1)} & \\
& \vec{q}_{n}
\end{array}\right]
$$

It is worth mentioning that when the only satellite from the second constellation is removed, the dual-GNSS constellations become a single constellation. In this case, the number of the unknown vector decreases from five to four, so the dimension of the design matrix $\overrightarrow{\boldsymbol{H}}_{(n-1)}$ becomes $(n-1) \times 4$. Although Equation (24) also describes the change of the design and weight matrices, it is different from Equation (12). On the basis of Equation (24), we can get

$$
\overrightarrow{\boldsymbol{H}}_{n}^{T} \overrightarrow{\boldsymbol{Q}}_{n} \overrightarrow{\boldsymbol{H}}_{n}=\left[\begin{array}{cc}
\overrightarrow{\boldsymbol{H}}_{(n-1)}^{T} \overrightarrow{\boldsymbol{Q}}_{(n-1)} \overrightarrow{\boldsymbol{H}}_{(n-1)}+\vec{q}_{n} \overrightarrow{\boldsymbol{h}}^{T} \overrightarrow{\boldsymbol{h}} & \vec{q}_{n} \overrightarrow{\boldsymbol{h}}^{T} \\
\vec{q}_{n} \overrightarrow{\boldsymbol{h}} & \vec{q}_{n}
\end{array}\right]
$$


The inverse of Equation (25) is given by

$$
\begin{aligned}
& \left(\overrightarrow{\boldsymbol{H}}_{n}^{T} \overrightarrow{\boldsymbol{Q}}_{n} \overrightarrow{\boldsymbol{H}}_{n}\right)^{-1} \\
& =\left[\begin{array}{cc}
\left(\overrightarrow{\boldsymbol{H}}_{(n-1)}^{T} \overrightarrow{\boldsymbol{Q}}_{(n-1)} \overrightarrow{\boldsymbol{H}}_{n-1}\right)^{-1} & \ldots \\
\ldots & \left(\vec{q}_{n}-\vec{q}_{n} \overrightarrow{\boldsymbol{h}}\left(\overrightarrow{\boldsymbol{H}}_{(n-1)}^{T} \overrightarrow{\boldsymbol{Q}}_{(n-1)} \overrightarrow{\boldsymbol{H}}_{(n-1)}+\vec{q}_{n} \overrightarrow{\boldsymbol{h}}^{T} \overrightarrow{\boldsymbol{h}}\right)^{-1} \vec{q}_{n} \overrightarrow{\boldsymbol{h}}^{T}\right)^{-1}
\end{array}\right]
\end{aligned}
$$

Consider Equation (10), and then we have

$$
\overrightarrow{\boldsymbol{R}}_{n}=\left[\begin{array}{l}
\overrightarrow{\boldsymbol{R}}_{(n-1)} \\
\left.\left(\vec{q}_{n}-\vec{q}_{n} \overrightarrow{\boldsymbol{h}}\left(\overrightarrow{\boldsymbol{H}}_{(n-1)}^{T} \overrightarrow{\boldsymbol{Q}}_{(n-1)} \overrightarrow{\boldsymbol{H}}_{(n-1)}+\vec{q}_{n} \overrightarrow{\boldsymbol{h}}^{T} \overrightarrow{\boldsymbol{h}}\right)^{-1} \vec{q}_{n} \overrightarrow{\boldsymbol{h}}^{T}\right)^{-1}\right]
\end{array}\right]
$$

with $\overrightarrow{\boldsymbol{R}}_{n} \in R^{5 \times 5}$ and $\overrightarrow{\boldsymbol{R}}_{(n-1)} \in R^{4 \times 4}$. From Equation (27), we can obtain

$$
\left\{\begin{array}{l}
\vec{r}_{n}(i)=\vec{r}_{(n-1)}(i), i=1, \cdots, 4 \\
\vec{r}_{n}(5)=\left(\vec{q}_{n}-\vec{q}_{n} \overrightarrow{\boldsymbol{h}}\left(\overrightarrow{\boldsymbol{H}}_{(n-1)}^{T} \overrightarrow{\boldsymbol{Q}}_{(n-1)} \overrightarrow{\boldsymbol{H}}_{(n-1)}+\vec{q}_{n} \overrightarrow{\boldsymbol{h}}^{T} \overrightarrow{\boldsymbol{h}}\right)^{-1} \vec{q}_{n} \overrightarrow{\boldsymbol{h}}^{T}\right)^{-1}
\end{array}\right.
$$

Therefore,

$$
\overrightarrow{P D O P}_{n}=\overrightarrow{P D O P}_{(n-1)}
$$

The equality in Equation (29) demonstrates that although removing one satellite reduces the dimension of the unknown vector, the PDOP keeps constant. Actually, this can also be understood from the point of the impact of different constellations on positioning calculation. If removing one satellite leads to a decrease in the number of unknown parameters, it means that there is only one satellite belonging to a certain constellation. The satellite is only utilised for determining the receiver clock bias relative to the corresponding constellation, and it has no influence on the positioning information directly linked with the PDOP. Therefore when the only satellite is removed, the PDOP neither increases nor decreases. This is different from the change of PDOP in Section 3.1.

Furthermore, according to the definition of TDOP in Equation (11), we have

$$
\left\{\begin{array}{l}
\overrightarrow{T D O P}_{(n-1)}=\sqrt{\vec{r}_{(n-1)}(4)} \\
\overrightarrow{T D O P}_{n}=\sqrt{\vec{r}_{n}(4)+\vec{r}_{n}(5)}
\end{array}\right.
$$

Similar to Equation (21), we define

$$
\Delta \overrightarrow{T D O P}_{n}=\overrightarrow{T D O P}_{n}^{2}-\overrightarrow{T D O P}_{(n-1)}^{2}
$$

as the variable quantity of the TDOP with the number of satellites. 
Combining Equation (30) with Equation (31), then the latter becomes

$$
\Delta \overrightarrow{T D O P}_{n}=\vec{r}_{n}(5)=\left(\vec{q}_{n}-\vec{q}_{n} \overrightarrow{\boldsymbol{h}}\left(\overrightarrow{\boldsymbol{H}}_{(n-1)}^{T} \overrightarrow{\boldsymbol{Q}}_{(n-1)} \overrightarrow{\boldsymbol{H}}_{(n-1)}+\vec{q}_{n} \overrightarrow{\boldsymbol{h}}^{T} \overrightarrow{\boldsymbol{h}}^{-1} \vec{q}_{n} \overrightarrow{\boldsymbol{h}}^{T}\right)^{-1}\right.
$$

According to the proof in our previous work (Teng and Wang, 2014), $\vec{r}_{n}(5)$ is positive. Thus,

$$
\overrightarrow{T D O P}_{n}>\overrightarrow{\operatorname{TDOP}}_{(n-1)}
$$

That is to say, if removing one satellite from the tracked dual-GNSS constellations reduces the dimension of unknown vector, the TDOP decreases. This is also different from the change of TDOP in Section 3.1.

As the PDOP and TDOP compose the GDOP, the equality in Equation (29) and the inequality in Equation (33) are the fundamental reasons for the change of the GDOP in our earlier studies (Teng and Wang, 2014).

3.3. Further Discussion on the Change of TDOP. In this section, we will further analyse the change of TDOP based on the expression of the variable quantity of the TDOP. The component and the monotonicity of the variable quantity of the TDOP are discussed, respectively.

3.3.1. The component of the variable quantity of the TDOP. For the convenience of discussion, the variable quantity of the TDOP in Section 3.1 and 3.2 can be summarised as

$$
\left\{\begin{array}{l}
\Delta T D O P_{n}=\left[q_{n}^{-1}+\boldsymbol{h} \boldsymbol{R}_{n} \boldsymbol{h}^{T}\right]^{-1} \sum_{i=4}^{5}\left[\boldsymbol{R}_{(n-1)}(:, i) \boldsymbol{h}^{T}\right]^{2} \\
\Delta \overrightarrow{T D O P}_{n}=\left(\vec{q}_{n}-\vec{q}_{n} \overrightarrow{\boldsymbol{h}}\left(\overrightarrow{\boldsymbol{H}}_{(n-1)}^{T} \overrightarrow{\boldsymbol{Q}}_{(n-1)} \overrightarrow{\boldsymbol{H}}_{(n-1)}+\vec{q}_{n} \overrightarrow{\boldsymbol{h}}^{T} \overrightarrow{\boldsymbol{h}}^{-1} \vec{q}_{n} \overrightarrow{\boldsymbol{h}}^{T}\right)^{-1}\right.
\end{array}\right.
$$

where

$$
\left\{\begin{array}{l}
\boldsymbol{R}_{(n-1)}=\left(\boldsymbol{H}_{(n-1)}^{T} \boldsymbol{Q}_{(n-1)} \boldsymbol{H}_{(n-1)}\right)^{-1} \\
\boldsymbol{R}_{n}=\left(\boldsymbol{H}_{n}^{T} \boldsymbol{Q}_{n} \boldsymbol{H}_{n}\right)^{-1}=\left(\boldsymbol{H}_{(n-1)}^{T} \boldsymbol{Q}_{(n-1)} \boldsymbol{H}_{n-1}+q_{n} \boldsymbol{h}^{T} \boldsymbol{h}\right)^{-1}
\end{array}\right.
$$

In Equation (34), we can conclude what $\triangle T D O P_{n}$ and $\Delta \overrightarrow{T D O P}_{n}$ have in common is using the weight and the vector of the removed satellite, and the weight matrix and the design matrix of the remaining satellites as variables. This point can be understood easily. And most of all, the significant difference between $\triangle T D O P_{n}$ and $\triangle \overrightarrow{T D O P}_{n}$ is that two items are required for $\triangle T D O P_{n}$. However, $\Delta \overrightarrow{T D O P}_{n}$ only needs one item.

The reason for this phenomenon can be explained on the basis of the definition of the TDOP. If removing one satellite from the tracked satellites cannot decrease the dimension of the unknown vector, there are two receiver clock biases (relative to the corresponding constellations) to be estimated. Correspondingly, the variable quantity of the TDOP $\left(\triangle T D O P_{n}\right)$ can be divided into two corresponding components, which refer to $\boldsymbol{R}_{(n-1)}(:, 4)$ and $\boldsymbol{R}_{(n-1)}(:, 5)$. 
In addition, if the dimension of the unknown vector decreases from five to four after one satellite is removed, the receiver clock bias (relative to the removed satellite) cannot be estimated anymore. The impact of this removed satellite on the TDOP can be regarded as the variable quantity of the TDOP. As a consequence, $\triangle \overrightarrow{T D O P} \vec{P}_{n}$ only includes one item.

3.3.2. The monotonicity of the variable quantity of the TDOP. The monotonicity of $\triangle \overrightarrow{T D O P}_{n}$ will be derived hereafter. We mainly derive the monotonicity with the weight of the removed satellite. Similar to the method in our previous work (Teng and Wang, 2014), we decompose $\overrightarrow{\boldsymbol{H}}_{(n-1)}^{T} \overrightarrow{\boldsymbol{Q}}_{(n-1)} \overrightarrow{\boldsymbol{H}}_{(n-1)}$ as

$$
\overrightarrow{\boldsymbol{H}}_{(n-1)}^{T} \overrightarrow{\boldsymbol{Q}}_{(n-1)} \overrightarrow{\boldsymbol{H}}_{(n-1)}=\boldsymbol{U} \boldsymbol{\Lambda} \boldsymbol{U}^{T}
$$

In Equation (37), $U$ is an orthogonal matrix, and $\boldsymbol{\Lambda}=\operatorname{diag}\left[\lambda_{1}, \cdots, \lambda_{4}\right]$ is a diagonal matrix. As $\overrightarrow{\boldsymbol{H}}_{(n-1)}^{T} \overrightarrow{\boldsymbol{Q}}_{(n-1)} \overrightarrow{\boldsymbol{H}}_{(n-1)}$ is a symmetric and positive definite matrix, the diagonal elements $\lambda_{i}(i=1 \sim 4)$ are positive (Horn and Johnson, 2010). After some complicated derivations, Equation (37) holds.

$$
\Delta \overrightarrow{T D O P}_{n}=\left(\vec{q}_{n}-\vec{q}_{n} \overrightarrow{\boldsymbol{h}}\left(\overrightarrow{\boldsymbol{H}}_{(n-1)}^{T} \overrightarrow{\boldsymbol{Q}}_{(n-1)} \overrightarrow{\boldsymbol{H}}_{(n-1)}+\vec{q}_{n} \overrightarrow{\boldsymbol{h}}^{T} \overrightarrow{\boldsymbol{h}}\right)^{-1} \vec{q}_{n} \overrightarrow{\boldsymbol{h}}^{T}\right)^{-1}=\frac{1+\gamma}{\vec{q}_{n}}
$$

where

$$
\gamma=\boldsymbol{v}^{T} \boldsymbol{\Lambda}^{-1} \boldsymbol{v}=\sum_{i=1}^{4}\left(\frac{v_{i}^{2}}{\lambda_{i}}\right)
$$

with $v_{i}(i=1 \sim 4)$ being the $i$ th element of the vector $\boldsymbol{v}=\sqrt{\vec{q}_{n}} \overrightarrow{\boldsymbol{h}} \boldsymbol{U}$.

To facilitate the derivation, by defining $\boldsymbol{\beta}=\overrightarrow{\boldsymbol{h}} \boldsymbol{U}=\left[\beta_{1}, \cdots, \beta_{4}\right]$, then we have

$$
\gamma=\sum_{i=1}^{4}\left(\frac{v_{i}^{2}}{\lambda_{i}}\right)=\vec{q}_{n} \sum_{i=1}^{4}\left(\frac{\beta_{i}^{2}}{\lambda_{i}}\right)
$$

Substituting Equation (39) into Equation (37) leads to

$$
\Delta \overrightarrow{T D O P}_{n}=\frac{1+\gamma}{\vec{q}_{n}}=\frac{1}{\vec{q}_{n}}+\sum_{i=1}^{4}\left(\frac{\beta_{i}^{2}}{\lambda_{i}}\right)
$$

In Equation (40), it is clear to see that the second item is irrelevant to the weight of the removed satellite $\left(\vec{q}_{n}\right)$. Therefore, for the case when removing one satellite from the tracked satellites does not reduce the dimension of the unknown vector, we can conclude that the variable quantity of the TDOP is monotonically decreasing with the weight of the removed satellite.

4. A NUMERICAL EXAMPLE. A numerical example is given to demonstrate the change of PDOP and TDOP in this section. For brevity, the weight matrix is assumed as an identity one.

Herewith we put emphasis on discussing the change of PDOP and TDOP in the case of the variant dimension of unknown vector. Suppose that there are five satellites in the 
dual-GNSS constellations, and only one satellite belongs to the second single constellation. Then the design matrix is given by

$$
\boldsymbol{H}_{5}=\left[\begin{array}{ccccc}
-0.2057 & 0.5397 & 0.8164 & 1 & 0 \\
0.5208 & -0.8430 & 0.1348 & 1 & 0 \\
-0.7745 & 0.3587 & 0.5210 & 1 & 0 \\
0.1494 & 0.7532 & 0.6406 & 1 & 0 \\
-0.9270 & 0.2141 & 0.3079 & 0 & 1
\end{array}\right]
$$

In this experiment, the fifth satellite is removed so that the four remaining satellites belong to the first constellation. The PDOP obtained from five satellites is the same as that obtained from the four remaining satellites. It is 5.6079 . However, before and after removing the fifth satellite, the TDOP decreases from $2 \cdot 9941$ to $2 \cdot 3452$. This example illustrates that when removing one satellite reduces the dimension of the unknown vector, the PDOP and TDOP may have different changes. Namely, the PDOP neither increases nor decreases, while the TDOP decreases.

5. CONCLUSIONS. From the point of the dimension of the unknown vector, this paper has theoretically derived the changes of PDOP and TDOP with the number of satellites in single point positioning with multi-GNSS constellations. The results have demonstrated that when the dimension of the unknown vector is invariant, the PDOP and TDOP always increase with the decrease in the number of satellites. However, if removing satellites leads to a decrease in the dimension of the unknown vector, the PDOP neither increases nor decreases. But, in this case, the change of the TDOP is different, and it becomes larger.

Moreover, the detailed expressions of the variable quantity of the PDOP and TDOP with regard to the number of satellites are also derived. Especially when removing satellites reduces the dimension of the unknown vector, the monotonicity of the variable quantity of the TDOP with the weight of the removed satellite is also taken into consideration. The theoretical derivation shows that the variable quantity of the TDOP is monotonically decreasing with the weight of the removed satellite. Such new characteristics about PDOP and TDOP, together with other new characteristics about GDOP in our former studies, can enrich the knowledge base on the DOP for multi-GNSS constellation-based positioning.

Besides the monotonicity of the variable quantity of the TDOP, the monotonicity of the variable quantity of the PDOP for multi-GNSS constellations will be considered in future investigations. In addition, other characteristics about the variable quantity of the PDOP and the TDOP (i.e., the contribution of different satellites to the variable quantity of the PDOP and the TDOP) also deserve further research.

\section{ACKNOWLEDGMENTS}

This work was supported by the National Natural Science Foundation of China (51277022) and the Fundamental Research Funds for the Central Universities (ZYGX2012J150). 


\section{REFERENCES}

Angrisano, A, Gaglione, S. and Gioia, C. (2013). Performance assessment of GPS/GLONGASS single point positioning in an urban environment. Acta Geodaetica et Geophysica, 48(2), 149-161.

Blanco-Delgado, N. and Nunes, F. (2010). Satellite selection based on WGDOP concept and convex geometry. 2010 5th ESA Workshop on Satellite Navigation Technologies and European Workshop on GNSS Signals and Signal Processing, Noordwijk, Netherlands.

Blomenhofer, H. (2004). GNSS in the 21st century. Acta Astronautica, 54(11), 965-968.

Dautermann, T. (2014). Civil air navigation using GNSS enhanced by wide area satellite based augmentation systems. Progress in Aerospace Sciences, 67(3), 51-62.

Defraigne, P. and Baire, Q. (2011). Combining GPS and GLONASS for time and frequency transfer. Advances in Space Research, 47(2), 265-275.

Hewitson, S. and Wang, J. (2006). GNSS receiver autonomous integrity monitoring (RAIM) performance analysis. GPS Solutions, 10(3), 155-170.

Horn, R. and Johnson, C. R. (2010). Matrix Analysis. Cambridge University Press.

Kaplan, D. and Hegarty, C. J. (2006). Understanding GPS Principles and Application. Artech House.

Li, B., Tan, Y. K. and Dempster, A. G. (2010). Using two global positioning system satellites to improve wireless fidelity positioning accuracy in urban canyons. IET Communications, 5(2), 163-171.

Rapinski, J., Cellmer, S. and Rzepecka, Z. (2012). Modified GPS/pseudolite navigation message. Journal of Navigation, 65(4), 711-716.

Rzepecka, Z., Rapinski, J., Cellmer, S. and Chrzanowski, A. (2014). Effect of additional distance measurement on satellite positioning. Acta Geodynamica et Geomaterialia, 11(1), 35-44.

Shi, H. L. and Cui, J.X. (2011). Where will satellite navigation be developed? Science China Physics, Mechanics \& Astronomy, 41(5), 539-546.

Teng, Y. L. and Shi, Y. B. (2012). Clock-based RAIM method and its application in GPS receiver positioning. Journal of Central South University, 19(6), 1558-1563.

Teng, Y. L. and Wang, J. (2014). New Characteristics of Geometric Dilution of Precision (GDOP) for MultiGNSS Constellations. Journal of Navigation, 67(6), 1018-1028.

Teunissen, P. J. G., Odolinski, P. and Odijk, D. (2014). Instantaneous Beidou+GPS RTK positioning with high cut-off elevation angles. Journal of Geodesy, 88(4), 335-350.

Torre, A. D. and Caporali, A. (2015). An analysis of intersystem biases for multi-GNSS positioning. GPS Solutions, 19(2), 297-307.

Wang, J. and Ober, P. B. (2009). On the availability of fault detection and exclusion in GNSS receiver autonomous integrity monitoring. Journal of Navigation, 62(2), 251-261.

Wang, J., N. Knight, N. and Lu, X. (2011). Impact of the GNSS time offsets on positioning reliability. Journal of Global Positioning Systems, 10(2), 165-172.

Xu, L. X., Li, X. H., Xue, Y. R., Cai, C. L. and Guo, M. J. (2012). System time offset based RAIM in combined GPS/Beidou navigation system. Proceedings of the 3rd China Satellite Navigation Conference (CSNC) 2012, Guangzhou, China.

Yang, Y. X., Li, J. L., Xu, J. Y., Tang, J., Guo, H. R. and He, H. B. (2011). Contribution of the Compass satellite navigation system to global PNT users. Chinese Science Bulletin, 56(26), 2813-2819. 